\title{
Tuning a Pentangle—A New Musical Vibrating Element
}

\author{
N. H. Fletcher \\ CSIRO Australia, and Research School of Physical Sciences and Engineering, \\ Australian National University, Canberra ACT 2601, Australia
}

(Received 18 March 1992; accepted 1 May 1992)

\begin{abstract}
$A B S T R A C T$
As part of a program in musical acoustics, it was required to design a nonregular but reflection-symmetric pentagonal gong, made from steel rod, of such geometry that the first 5 or 6 of its planar normal modes provide partial tones in nearly harmonic relationship, in order to achieve a musically satisfactory bell-like sound. Techniques are described for exploring the accessible configuration space to reach this objective, using a combination of analytic solution and finite-element analysis. The results and methods, which may be of wider applicability and interest, are described in detail, as also are two independent solutions reached to the design problem. The final gong has markedly different tone quality depending upon whether it is struck so as to excite the harmonic in-plane modes or the inharmonic outof-plane modes.
\end{abstract}

\section{INTRODUCTION}

This paper reports upon an interesting problem relating to adjustment of the mode frequencies of a multiply bent rod in such a way that it can be used as a tuned musical instrument of the percussion family. The discussion throws some light upon both the behaviour of mode frequencies and the factors that are of importance in musical percussion instruments.

Applied Acoustics 0003-682X/93/\$06.00 (c) 1993 Elsevier Science Publishers Ltd, England. Printed in Great Britain 
The problem arose during the course of development of a family of new tuned percussion instruments by Australian composer, and CSIRO Artist-in-residence, Moya Henderson. The first instrument of this series, the Alemba, ${ }^{1,2}$ used a set of tuned triangles, each of which was coupled to a tube resonator through a driven diphragm as shown in Fig. 1. By tuning each resonator to the frequency of the second mode of the triangle to which it is coupled, the instrument was made to have a sustained and mellow sound, the exact timbre of which could be adjusted by using beaters of different mass and hardness. The instrument has been used in orchestral performances.

The triangles used in the Alemba are of isosceles shape, with an open apex as in a standard orchestral triangle. The relation between the mode frequencies of such a structure can be varied to some extent by changing the base angles and the curvature of its corners, and this has been investigated in a study by Dunlop. ${ }^{3}$ The nominal pitch of the triangle can be fixed by its overall size. In this way two other mode frequencies can be brought into harmonic relation with the nominal pitch. The remaining inharmonic partials are, however, quite prominent, and this is regarded by musicians as a limitation to the usefulness of the instrument.

The next instrument in the series, called the Tosca Bells, adopted a symmetrical pentagonal vibrating element, open at one corner, of the general shape shown in Fig. 2. This shape was chosen on aesthetic, rather than acoustic grounds, and clearly the motivation is its bell-like profile. As with the Alemba, it was intended to couple a set of these elements to appropriately tuned pipe resonators. The first 'pentangle',

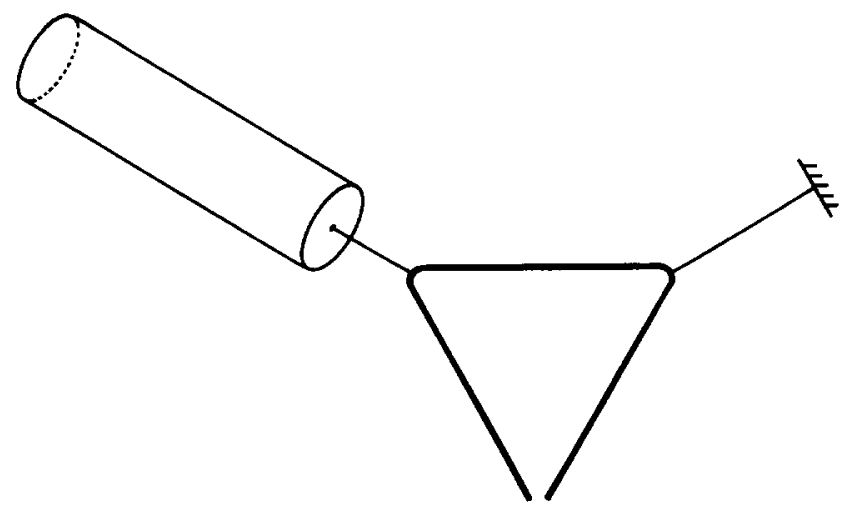

Fig. 1. The sound-producing elements of the Alemba are a set of metal rods, bent to triangular shape and coupled to separate tubular resonators, each tuned to the second mode of the associated triangle. The coupling is to a flexible membrane, covering one end of each tube, through a taut cord. The triangles are excited by blows from soft hammers. 
constructed on these aesthetic grounds, did in fact produce a sound with several partials in nearly harmonic relation, but prominent discordant partials were also clearly audible. The present study was undertaken in an attempt to produce a design with this general shape and having a set of partials with as nearly harmonic a relationship as possible.

Development of a pentangle of this type is in many ways analogous to bell-founding practice, ${ }^{4,5}$ in which the appropriate shape has been developed through centuries of experience. In the making of bells, which represent a large investment, it is normal practice in most countries to refine the initial mode frequencies of the cast bell to the desired values by turning metal off the inside of the bell according to carefully established rules. In the case of a pentangle bent to well-defined geometry from uniform metal rod or bar, we might hope that this tuning step could be eliminated in the interests of simplicity and economy.

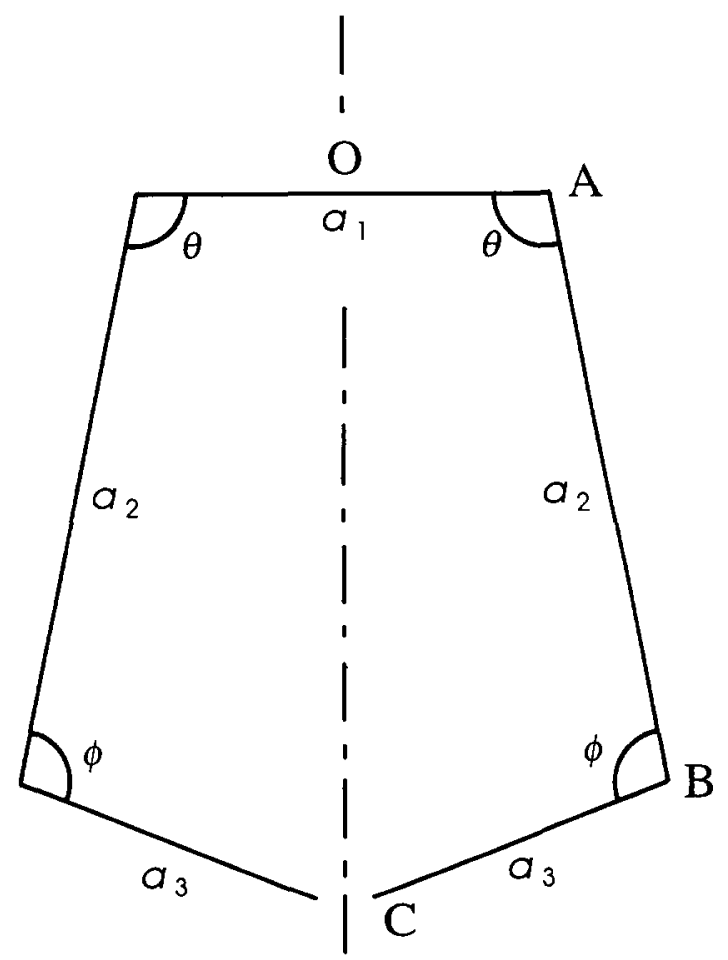

Fig. 2. The shape of a simplified Tosca Bell pentangle. The pentangle has an axis of symmetry, and the independent dimensional parameters $a_{1}, a_{2}, a_{3}, \theta$ and $\phi$ are shown. In a real pentangle, the corners cannot be ideally sharp, and the radius of curvature $r$ of the bends appears as a further parameter. 


\section{CALCULATION OF MODE FREQUENCIES}

In order to attack this problem, we clearly require an accurate means for calculating the normal mode frequencies of a multiply bent rod. We will require to optimise these frequencies, which means that the calculation will be inside some sort of iteration loop, so that there is a high priority on speed and efficiency. This latter condition places finite-element packages, which are generally designed as general-purpose tools, at something of a disadvantage relative to a purpose written program. As we see below, an optimal approach involves using both these methods.

Detailed solution of the mode-frequency problem for a rod of appreciable diameter is a very difficult undertaking, so that an initial simplification is highly desirable. The preferred construction material for the pentangles is $12.7 \mathrm{~mm}$ diameter mild steel rod, since it is not expensive, is easily worked, and has appropriate vibrational properties as far as internal damping and the mechanical admittance of the finished article is concerned. The length of rod in an individual pentangle varies from about 0.5 to $1.5 \mathrm{~m}$, so that the rod can be regarded as moderately thin. This suggests that a thinrod approximation is close to validity. This is the usual approximation for the behaviour of beams that is implemented in finite-element packages. The next simplification arises because the instrument is designed to be played using a hammer blow with velocity only in the plane of the pentangle. Ideally such an impulse will excite only modes lying in the plane and, even in non-ideal cases, the amplitudes of these in-plane modes will be much greater than those of the non-planar modes. A calculation limited to plane deflections is thus a good starting point, and may well be entirely adequate for practical cases.

Even with these simplifications, however, a typical finite element solution takes a moderate amount of computer time, since a large number of elements must be used to obtain accurate frequencies for the higher modes. It is therefore worthwhile to make one further simplification for an initial survey, and this is to approximate the corners as being ideally sharp. At one stroke this eliminates one parameter, the corner curvature, and allows an analytic solution for the straight-rod segments, which can be joined by appropriate boundary conditions at the corners. Implementation of this analytic solution is outlined in the Appendix. A computer program to evaluate the mode frequencies is simple to write, gives completely accurate eigenvalues, and executes much more rapidly than the finite-element package on a microcomputer, thus allowing a rapid survey of the primary parameter space for the shape of the pentangle.

The results of a calculation using this analytic approach are given in Fig. 3, which shows the variation of the first 6 mode frequencies as a $1 \mathrm{~m}$ 


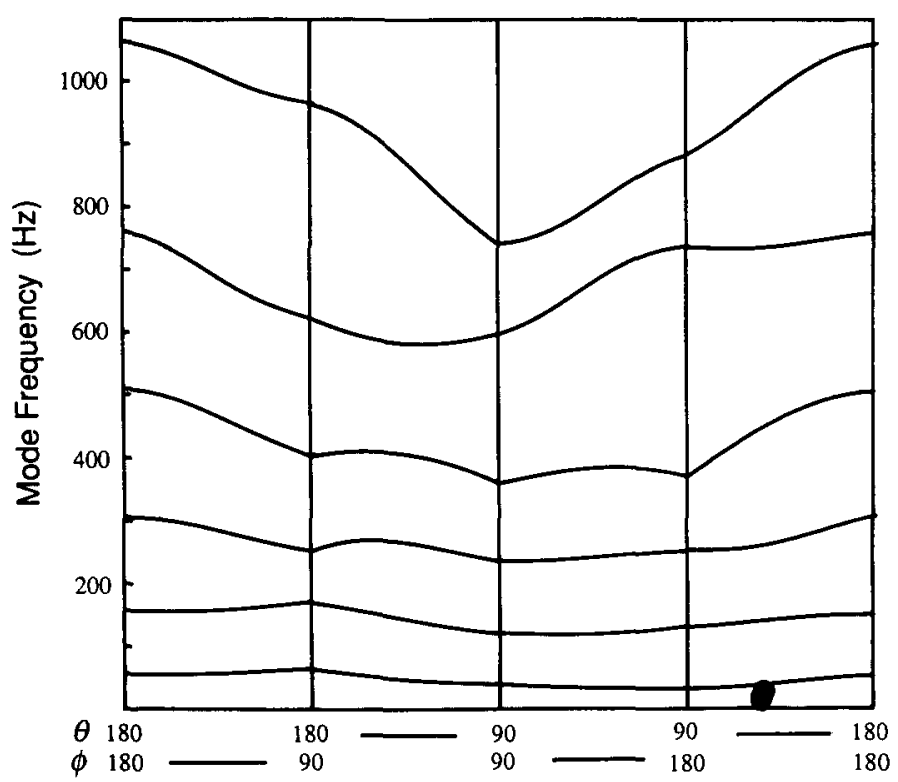

Fig. 3. Variation of the frequencies of the first few in-plane modes for a thin rod as it is bent into a pentangular shape. Angles $\theta$ and $\phi$ are as defined in Fig. 2, and side lengths are chosen so that $R_{21}=a_{2} / a_{1}=2, R_{31}=a_{3} / a_{1}=1$.

length of $12.7 \mathrm{~mm}$ diameter steel rod is progressively bent into the shape of a rectangular pentangle and then unbent in the opposite order. Clearly there are large changes in the relative frequencies of the modes, which gives some hope that a shape might be achieved to give a nearly harmonic relationship between some appreciable number of them.

Once a suitable shape has been determined to first order, using this analytic approximation and the tuning philosophy outlined in the next section, it is relatively straightforward and not too time-consuming to use a finite-element package to refine the shape by including the finite curvature of the corners.

\section{TUNING PHILOSOPHY}

Restricting ourselves to symmetrical shapes and leaving aside the possibility of changing the curvature at the bends, it is clear from Fig. 2 that we have 5 parameters $\left(a_{1}, a_{2}, a_{3}, \theta, \phi\right)$ at our disposal for tuning the bell. This suggests that we should aim to tune 5 modes or, put more usefully, a basic pitch and 4 mode-frequency ratios relative to it. This is essentially the number of modes explicitly tuned in a church bell or carillon bell. ${ }^{6}$ For a given shape, the basic pitch is determined by the overall size 
$a_{1}+2 a_{2}+2 a_{3}$, so that it is convenient to take the parameter set to be $\left(R_{21}, R_{31}, \theta, \phi\right)$ where $R_{21}=a_{2} / a_{1}$ and $R_{31}=a_{3} / a_{1}$.

Since the time of Pythagoras, 2500 years ago, it has been known that musical notes sound pleasantly together if their frequencies are in the ratio of two small integers. The psychophysical basis for this observation has been subject to detailed investigation ${ }^{7,8}$ since the initial work of Helmholtz a century ago, and the effect probably arises in large measure from the regularly repetitive nature of the waveforms of such sounds, though the matter is complex. Musical instruments with sustained tones do in fact produce such precisely repetitive waveforms as a result of mode locking caused by the nonlinearity of their excitation mechanism, ${ }^{9}$ though the same thing is achieved only approximately in instruments with transient excitation as in the guitar or the piano.

It must be realised, however, that the audible effect of pure tones sounding in combination, whether these are produced artificially or as radiation from the normal modes of some solid vibrator, is quite different from the effect produced when two complex tones are sounded together, each having its own set of harmonics. In the former case no beats are produced, unless the frequencies of two normal modes lie fortuitously close together, while in the latter there may be beats between any pair of upper partials whose frequencies are comparable. The tuning tolerance acceptable for natural bell-like sounds is thus much wider than that for bell-like chords produced by sounding the component notes on other instruments.

In percussion instruments of the bell or gong family, the sound can be listened to in two ways. In holistic listening, the perception is of a welldefined musical pitch and a characteristic musical timbre or tone-quality; in analytical listening, the perception is of the set of individual partials making up the sound. For a successful musical instrument, the relation between the partials has to be such as to encourage holistic listening, and this is most readily achieved if the most prominent partials have frequencies in integral (harmonic), or nearly integral, frequency relationship.

Such a relationship can be written as a product of primes $2^{n} 3^{m} 5^{s} \ldots$ where $n, m$ and $s$ are small positive or negative integers. The degree of accuracy of the required tuning is highest if only the factor 2 is involved (octaves), fairly critical if both 2 and 3 occur (fifths and fourths) and much less critical if 5 occurs as well (major and minor thirds). Tuning of intervals involving 7, or higher primes, is very uncritical as far as consonance is concerned. The exact sequence of partial tones in the sound, and their relative strengths, has a great bearing on the sound quality, as does also, indeed, the strength and general frequency distribution of the untuned higher partials which are generally not heard analytically. 
TABLE 1

Harmonic or 'Just' Pitch Ratios (First line relative to $C_{1}$, second line relative to $C_{3}$ )

\begin{tabular}{cccccccccccccc}
\hline $\mathrm{C}_{1}$ & $\mathrm{E}_{1}$ & $\mathrm{~F}_{1}$ & $\mathrm{G}_{1}$ & $\mathrm{C}_{3}$ & $\mathrm{E}_{3}$ & $\mathrm{G}_{3}$ & $\mathrm{C}_{4}$ & $\mathrm{E}_{4}$ & $\mathrm{G}_{4}$ & $\mathrm{C}_{5}$ & $\mathrm{E}_{5}$ & $\mathrm{E}_{5}$ & $\mathrm{G}_{5}$ \\
1 & $5 / 4$ & $4 / 3$ & $3 / 2$ & 4 & 5 & 6 & 8 & 10 & 12 & 16 & $96 / 5$ & 20 & 24 \\
0.25 & 0.3125 & 0.3333 & 0.375 & 1 & 1.25 & 1.5 & 2 & 2.5 & 3 & 4 & 4.8 & 5 & 6 \\
\hline
\end{tabular}

For convenience, Table 1 sets out the harmonic (or 'just') frequency ratios for the musical pitches with which we shall be concerned here. The subscripts to pitch symbols refer to the octave in which they occur, $\mathrm{C}_{4}$ being 'middle $\mathrm{C}$ ' and $\mathrm{C}_{1}$ the lowest $\mathrm{C}$ on the piano keyboard. The notes of a keyboard instrument, such as the piano, are tuned to 'equal temperament', in which all the fifths are flattened (tempered) by about 0.1 percent so that all 12 notes of the scale have the same frequency ratio $2^{1 / 12}$ to their neighbours. This results in major and minor thirds which differ from the harmonic ratios $5 / 4$ and $6 / 5$ by about 1 percent.

To produce a satisfying bell-like sound we aim to tune at least 4 prominent partials into small-integer ratios with the particular partial tone-generally the strongest low partial - that is taken as the nominal pitch of the bell. If the aim is to produce a church-bell-like sound, then it also turns out to be highly desirable to include a minor-third interval ( $6: 5$ or one of its octaves) relative to this nominal.

For a straight rod of radius $a$ and length $L$ with free ends, the frequency of the $n$th mode is given approximately by

$$
f_{n} \approx A \frac{a}{L^{2}}\left(n+\frac{1}{2}\right)^{2}
$$

where $A$ is a constant depending upon the density and Young's modulus of the rod material. The frequencies of the modes thus have ratios close to a sequence which can be most helpfully written $0.36: 1.00: 1.96: 2.56: 3.24 \ldots$. The lowest frequency is well removed from the others and is not radiated very efficiently, so that it is natural to take the frequency of the second mode as defining the nominal pitch. From Fig. 3 we see that the lowest mode of a bent rod is similarly isolated in relative frequency from the upper modes, so that we make the same nominal pitch assignment for the Tosca Bell. It is appropriate to note that the second mode is also generally taken as defining the pitch of a church bell, the first mode being called the hum or undertone.

Our task is now to explore the 4-dimensional parameter space $\left\{R_{21}\right.$, $\left.R_{31}, \theta, \phi\right\}$ and find configurations for which the frequency ratios, relative to the second mode as nominal, have the required simple form. This task is potentially very extensive numerically, but it can be simplified greatly 
by proceeding one mode at a time and by adding parameters one at a time, as we now show.

The sub-nominal first mode frequency is of little importance, since the resonator in the complete Tosca instrument can be tuned to the second mode and will then radiate little at this lower frequency. We therefore neglect it and look only at the higher mode frequencies relative to mode 2. If we assume reasonable values for two of the parameters, say the sidelength ratios $R_{21}$ and $R_{31}$, then we can explore numerically the 2-parameter $\{\theta, \phi\}$ configuration space by computing mode frequencies over a grid of about $5 \times 5$ points and drawing $(\theta, \phi)$ contours along which the required harmonic frequency relationships are met. The base plane of Fig. 4 shows an example of such contours for modes 3 and 4 at acceptable frequency ratios such as $2: 1$ or $3: 1$ relative to mode 2 . If a solution exists, then these two contours must cross at a point $\mathrm{A}$ within the accessible $\{\theta, \phi\}$ space.

We now extend the phase space to three dimensions by calculating a similar set of acceptable $(\theta, \phi)$ contours for additional values of one of the remaining parameters, say $R_{21}$. This allows us to draw surfaces in the 3-dimensional $\left\{\theta, \phi, R_{21}\right\}$ space corresponding to acceptable values of

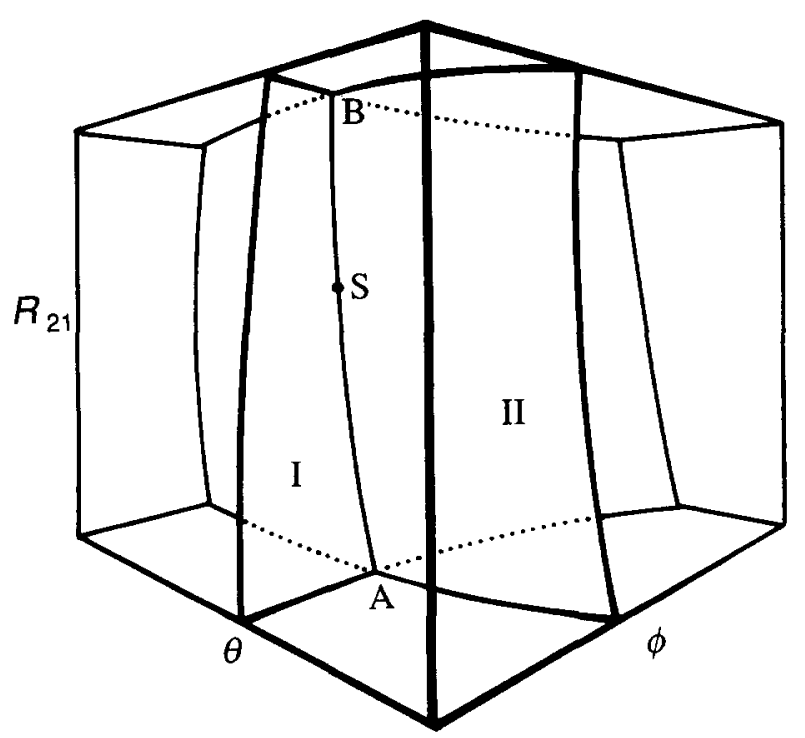

Fig. 4. Solution surfaces in a 3-dimensional configuration space. Surface I corresponds to a satisfactory ratio of the frequency of mode 3 to that of mode 2 , and surface II to a satisfactory ratio for mode 4 . These two surfaces intersect in a curve $A B$. If the solution surface giving a satisfactory ratio for mode 5 intersects $\mathrm{AB}$ in a point such as $\mathrm{S}$, then the configuration represented by $S$ is a satisfactory solution for modes 3,4 and 5 relative to mode 2. 
the frequency ratios for modes 3 and 4, as shown in Fig. 4. These two surfaces will intersect in a curve $\mathrm{AB}$, if a solution indeed exists.

We can draw a third surface in Fig. 4 corresponding to an appropriate ratio for the frequency of mode 5 . If this surface cuts the solution curve $A B$ for modes 3 and 4 , for example at the point $S$, then this point represents a satisfactory solution for modes 3,4 and 5 relative to mode 2 . The process can then be continued by including the remaining parameter, extending point $\mathrm{S}$ into a curve, and seeking an intersection with the surface for mode 6 at an acceptable frequency ratio. The advantage of this procedure is that it limits the amount of configuration space that must be explored at each step to that near a previously established curve or surface, and thus greatly reduces the calculation time involved.

In fact it is an effective procedure, once the approximate location of the solution point in configuration space has been identified, to search for the solution iteratively in the orthogonal $\{\theta, \phi\}$ and $\left\{R_{21}, R_{31}\right\}$ subspaces in turn. Thus Fig. 5 shows such a section in $(\theta, \phi)$ space for $R_{21}=2$, $R_{31}=1$, which is close to, but not coincident with, the exact solution ratio. It is clear that there are three regions in this sub-space, marked $S_{I}$, $\mathrm{S}_{\mathrm{II}}$ and $\mathrm{S}_{\mathrm{III}}$, which are close to multiple intersections of the individual solution surfaces for particular modes. Exploration can then be limited to the immdediate vicinity of these regions.

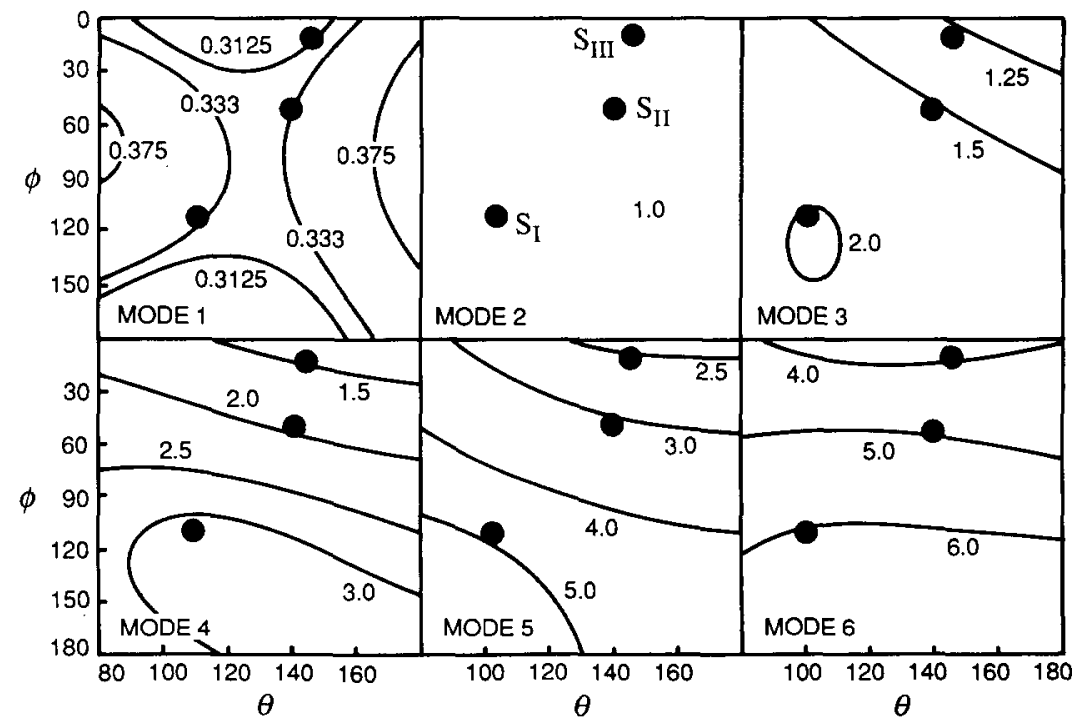

Fig. 5. Contours in the $\{\theta, \phi\}$ sub-space with $R_{21}=2, R_{31}=1$ for modes 1 to 6 of a pentangular frame. Regions near $S_{I}, S_{I I}$ and $S_{I I I}$ are close to possible solutions, which can be approached by successive optimisation in $\{\theta, \phi\}$ and $\left\{R_{21}, R_{31}\right\}$ sub-spaces. 


\section{SOLUTIONS}

Using the methods outlined in the previous section, these solutions were refined for the idealised sharp-corner tuning problem. Other useful solutions may possibly exist for greatly different side-length ratios, since the exploration was not completely exhaustive, but from a practical point of view it was satisfying to find even one! In fact only the solutions associated with region I turn out to be essentially exact. In the case of regions II and III the solution surfaces do not all pass through the solution point, but simply approach closely to it. Details of the initial solutions are given in Tables 2 and 3.

Solution I bears a fairly close resemblance to the original Tosca Bell configuration, designed on purely aesthetic grounds, except that the top angles are closed so much that the two free ends overlap. This is unfortunate, but simply means that the frame must be bent slightly out of plane so that there is adequate clearance. Modes 2 to 6 can be tuned exactly, in the sharp-corner approximation, and give a well-spread set of modes, including a minor third at mode 5. The nominal pitches of these notes are indicated, taking the nominal pitch of the bell as a whole to be tenor-C $\left(\mathrm{C}_{3}\right)$. The sub-nominal mode 1 has an unfortunately dissonant pitch close to $\mathrm{F} \#_{2}$, but this can be ignored, as we discussed above.

Solution II gives a bell of 'coat-hanger' shape which, from Table 3, has a well distributed set of mode frequencies. The sub-nominal has, in this case, a well-located near-harmonic frequency. Once again there is a minor third, this time at mode 6 and, since mode 3 has frequency ratio 1.5 , there may be an implied fundamental at frequency ratio 0.5 , an octave below the nominal pitch, for psychophysical reasons.

Solution III has a very flattened shape because of the small value of $\phi$. The mode frequencies are densely clustered in the range 1.0 to 2.5 and contain no fewer than three major thirds relative to the nominal pitch. The subjective pitch may again be below the nominal pitch because of the close spacing of these mode frequency ratios. The shape of this pentangle, however, is not satisfactory for practical reasons, particularly when we

TABLE 2

Initial Configurations

\begin{tabular}{lcccc}
\hline & $R_{21}$ & $R_{31}$ & $\theta(\mathrm{deg})$ & $\phi(\mathrm{deg})$ \\
\hline Solution I & 1.95 & 0.92 & 96 & 93 \\
Solution II & 2.20 & 1.07 & 135 & 46 \\
Solution III & 1.85 & 0.97 & 146 & 10 \\
\hline
\end{tabular}


come to consider rounded corners. For this reason this solution was not carried any further.

As the final important refinement to the design problem, the finite-element package Strand $5^{10}$ was used, again on an AT-compatible microcomputer, to include the effects of corner rounding. This package is particularly suitable for the calculation since its structure allows access to all the files and executable modules, so that it was easy to write a batch program to perform the necessary exploration of configuration space in the immediate vicinity of the initial solutions. It also does not require inclusion of external constraints on the pentangle. Optimisation, using the approach described above, typically takes only a few hours.

It is not very useful to report the calculated effects of changing bend radius upon mode frequencies, since this depends significantly upon which other parameters are kept constant. Nominally the other parameters are the side-length ratios, but when corner curvature is included we must define exactly how these are to be measured - between centres of curvature, between the intersections of the axes of the rod segments, or in some other way. The choice will have a significant influence upon the results when the corner radii are large or the bend angles large.

For steel rod, the minimum reasonably achievable bend radius corresponds to bending the rod around itself, giving a neutral-section bend radius about equal to the rod diameter, so that corrections to straightside lengths of at least this magnitude are involved. Since the rod diameter introduces an absolute scale into the problem, it is necessary to design the highest pitched gong to have at least the minimum achievable bend radius, after which the lower pitched gongs can be derived by simple scaling. The relative sharpness of the bend clearly depends upon the pitch of this smallest gong, and various design solutions are possible. In one particular case, the calculation was based upon using $12.7 \mathrm{~mm}$ steel rod and an internal bend radius of $20 \mathrm{~mm}$ (neutral section bend radius $26 \mathrm{~mm}$ ) at a rod length of $1000 \mathrm{~mm}$. This allowed design of a set of bells covering pitches up to about $\mathrm{C}_{4}$, middle $\mathrm{C}$, without impractically sharp

TABLE 3

Initial Mode Frequency Ratios

\begin{tabular}{lcccccc}
\hline Solution I & $(0.35)$ & 1.00 & 2.00 & 3.00 & 4.80 & 6.00 \\
Pitches & $\left(\mathrm{F}_{1}\right)$ & $\mathrm{C}_{3}$ & $\mathrm{C}_{4}$ & $\mathrm{G}_{4}$ & $\mathrm{~Eb}_{5}$ & $\mathrm{G}_{5}$ \\
Solution II & $(0.32)$ & 1.00 & 1.51 & 1.98 & 2.99 & 4.80 \\
Pitches & $\left(\mathrm{E}_{1}-\mathrm{F}_{1}\right)$ & $\mathrm{C}_{3}$ & $\mathrm{G}_{3}$ & $\mathrm{C}_{4}$ & $\mathrm{G}_{4}$ & $\mathrm{~Eb}_{5}$ \\
Solution III & $(0.31)$ & 1.00 & 1.25 & 1.46 & 2.49 & 4.04 \\
Pitches & $\left(\mathrm{E}_{1}\right)$ & $\mathrm{C}_{3}$ & $\mathrm{E}_{3}$ & $\mathrm{G}_{3}$ & $\mathrm{E}_{4}$ & $\mathrm{C}_{5}$ \\
\hline
\end{tabular}


bends. With this assumption, Solutions I and II were refined to produce practical gong designs, as shown in Fig. 6.

In the case of Solution I, rounding of the corners requires only small adjustments to the straight-side lengths and reduction in the inside angles to approach again the design mode frequencies to an accuracy better than 2 percent. The shape of this final Tosca Bell is essentially rectangular, with a large side overlap, as shown in the lower part of Fig. 6. In the case of Solution II, however, rounding of the lower corners produces a considerable change in mass distribution, as can be seen in the upper part of Fig. 6. This necessitates a considerable reduction of the angle $\phi$ relative to the sharp-corner configuration. Nevertheless, the original calculated mode frequencies are regained to better than 1 percent. The extent of this angular change, however, supports the view that a pentangle corresponding to Solution III could probably not be made by simple bending of the rod.

By relaxing the planar constraints applied to the finite-element solution, it is simple to calculate the frequencies of the out-of-plane modes as well as of the planar modes in which we are primarily interested. The Strand5 package also allows visualisation of these modes. We shall not follow up this extension here, as its practical importance is small. The existence of

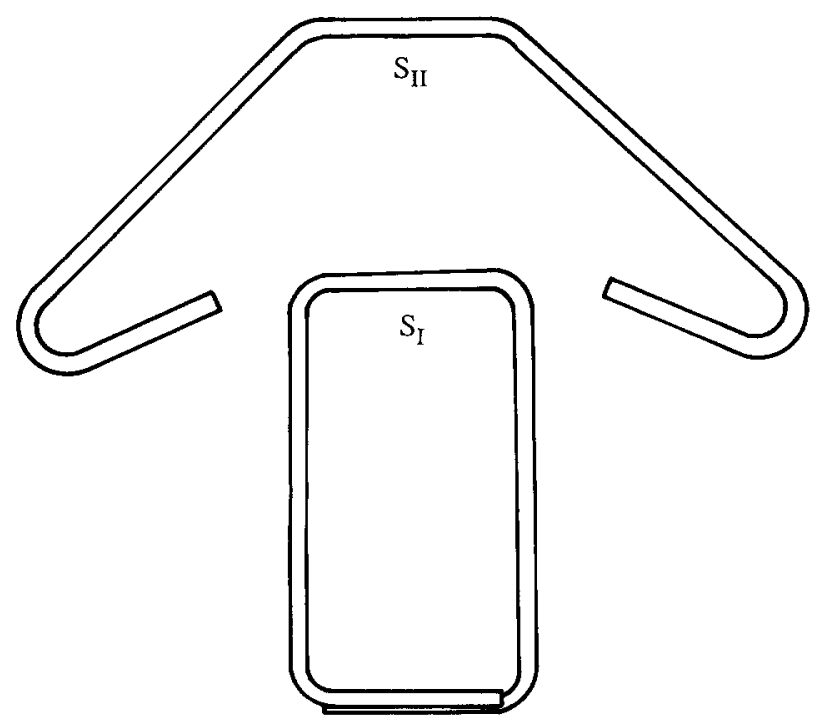

Fig. 6. Shapes of the two Tosca Bells derived from optimisation of solutions near regions $S_{1}$ and $S_{1 I}$ of Fig. 5, using a finite-element program to include corner bend radii. In the case of Solution $I$, the pentangle is bent slightly out of plane to give clearance between its two end sections. 
these inharmonic out-of-plane modes, however, allows to the performer a degree of control of the timbre of the instrument, since it can be struck so as to either minimise or maximise the amplitude of these modes relative to the harmonic in-plane modes.

The pentangles, as designed above, require no hand-tuning, their mode frequencies being defined by their basic shape. The same is true to some extent of traditional church bells, but it is almost universal practice to fine-tune the mode frequencies by turning small amounts of metal off the interior surface of the bell on a lathe, following recipes which have been established by long experience. Clearly the same sort of procedure could be used with pentangles, both to reduce the residual tuning discrepancies of the first six modes and perhaps also to tune some of the higher modes. A practical approach to this tuning problem for the case of bells has been developed by Mills. ${ }^{11}$ This involves evaluating the effect on the tuning of all modes of the removal of a small amount of metal from each of a large number of bands along the interior surface of the bell. A linear programming procedure is then used to define a metal-removal schedule that will produce optimal tuning. Such a procedure is readily implemented for a pentangle by using the finite-element package to determine the effect of filing metal off the rod in various locations. We have not, however, attempted to carry out this fine-tuning.

\section{SCALING}

Once a suitable design has been achieved for a pentangle element of some assumed size, it is necessary to scale this design to the size necessary to produce the required set of nominal pitches for the complete instrument. There are three possible approaches.

Conceptually the most straightforward is simply to scale all the dimensions of the pentangle (rod length, diameter and bend radius) uniformly. From eqn (1), the mode frequencies will then all vary inversely with the scale factor. The practical difficulty is that this requires a different rod diameter and bend radius for each pentangle of the set.

At the other extreme, one might keep both the rod diameter and bend radius constant and scale only the lengths of the rod segments. This would require that we perform a separate finite-element optimisation to incorporate corner rounding for each different member of the set, and the limitations imposed by a fixed bend radius might mean that the overtone structure of the pentangles might have to change at certain points along the compass. This is not very satisfactory.

Most practically appealing, therefore, is to use the same diameter rod 
for all pentangles, scaling rod length and bend radius, and to use once more the scaling law eqn (1), which shows that the mode frequencies in this case vary inversely as the square of the scale factor. This has economic advantages, though a different bending die has to be made for each size of pentangle.

\section{RESULTS}

Several sizes of members of the Tosca Bell family, corresponding to the refined versions of Solutions I and II, have been made. It is necessary to evaluate these both from the viewpoint of agreement with the calculated design and also, more importantly, for the musical effectiveness of their sound.

Table 4 shows the measured mode frequency ratios of two Tosca Bells, constructed to be as close as possible to the calculated curved-corner designs. The measured deviations from the calculated frequencies can be ascribed in large measure to small deviations from the desired geometry of the pentangle, since the rod was bent by hand in a simple jig. Agreement is certainly adequate to validate the design principles.

Also shown in Table 4 are the frequencies of the out-of-plane modes, which form an inharmonic series interlacing those of the planar modes. In practice, and despite the lack of planarity of the pentangle made necessary by overlap of the ends in Solution I, it is easily possible to execute the strike so as to excite almost exclusively the in-plane or the out-of-plane modes. The large inharmonicity of the out-of-plane modes creates a very different sound, and thus places some interesting effects in the hands of the performer.

In judging musical effectiveness, we can either take the view that we are attempting to simulate the sound of a traditional western European

TABLE 4

Calculated and Measured Frequency Ratios

\begin{tabular}{lcccccc}
\hline & \multicolumn{7}{c}{ Mode numbers } \\
& $\mathbf{1}$ & $\mathbf{2}$ & $\mathbf{3}$ & $\mathbf{4}$ & $\mathbf{5}$ & $\mathbf{6}$ \\
\hline Design Solution I & 0.35 & 1.00 & 1.96 & 3.05 & 4.82 & 6.13 \\
Pentangle I (measured) & $\mathbf{0 . 3 5}$ & 1.00 & 2.01 & 3.05 & $\mathbf{4} \cdot 79$ & 5.93 \\
Out-of-plane (measured) & 0.39 & 1.43 & 2.40 & $?$ & 4.97 & 9.57 \\
Design Solution II & 0.32 & 1.00 & 1.50 & 1.99 & 3.04 & 4.76 \\
Pentangle II (measured) & 0.33 & 1.00 & 1.49 & 1.96 & 3.05 & 4.75 \\
Out-of-plane (measured) & 0.83 & 1.06 & 1.42 & 2.57 & 4.03 & 5.17 \\
\hline
\end{tabular}


church bell, or we can simply require that the sound be pleasant, a matter which can be judged only subjectively.

For a traditional church bell, ${ }^{6}$ the first five modes are the hum or undertone, with frequency $1: 2$ relative to the second mode, the fundamental or prime, which is the reference frequency, the tierce or minor third $(6: 5)$ and the nominal or octave $(2: 1)$. Clearly neither of the two Tosca Bells comes close to this set of frequencies. Solution I contains the correct frequency ratios, including the minor third, to within powers of 2, except in the case of the first mode, but they are spread over several octaves rather than being concentrated. Solution II has rather more closely clustered mode frequencies and again has a minor third. Solution III has mode frequencies clustered more like those of a church bell but, as explained above, this design has not been followed up for practical reasons. Musical effectiveness cannot therefore be a matter of exact simulation but must rather rely on production of an appropriate subjectively belllike sound.

Since the diameter of the rod is very small relative to the wavelength of the lowest modes, these are radiated very inefficiently and can be heard, in the sound from an isolated pentangle, only in the near field. The radiated sound is thus dominated by the inharmonic higher modes. Attachment of a simple bucket-shaped non-resonant radiator (in fact a plastic bucket was used) by means of a short cord to one of the corners of the pentangle, however, increases the radiation efficiency of the lower modes very greatly, while not adding much to the radiated intensity of higher modes, which are attenuated in the cord and in the resonator material. Such an arrangement is a first approximation to the structure of the completed Tosca Bell instrument. The sound is judged subjectively as being mellow and pleasant, which was the original aim of the design exercise.

In order to evaluate the musical effectiveness of the design, we have now constructed a complete chromatic octave (13 notes) of the Type II bells, again using simple hand jigs. Frequency measurements for all the in-plane modes shows that the fundamental of each bell is within about 1.5 percent of its nominal value, and the mode tuning of each bell agrees with the design to better than 2 percent. These bells are to be coupled to a carefully designed sound-board radiator, rather than to individual tubular resonators, to produce a complete musical instrument. The coupling itself requires analysis, taking account of the mechanical impedance of the soundboard. The ideal attachment point should be near to a node for the musically important modes, in order that their tone may be sustained, and fortunately the sharp bends are close to ideal for this purpose. The compliance of the coupling cord itself provides a desirable high-frequency 
cut-off behaviour and reduces the prominence of inharmonic upper partials.

Preliminary experience suggests that these pentangular vibrating elements will provide the basis for an interesting new musical tuned-percussion instrument, with a mellow bell-like basic sound quality but with freedom allowed to the performer to introduce inharmonic components at will. The design method developed can be applied to bars of non-circular cross-section, in order to vary the relationship between in-plane and outof-plane modes, or to elements of more complex geometry.

\section{ACKNOWLEDGMENTS}

The author is most grateful to Moya Henderson for posing this problem, to Michael Coulthard for his preliminary work using finite-element methods, and particularly to Paul Drew for constructing and measuring the final Tosca Bells.

\section{REFERENCES}

1. Henderson, M., The discovery of a new musical sound. Bull. Australian Acoust. Soc., 12(1), (1984) 9-11.

2. Dunlop, J. I., The acoustics of the alemba. Bull. Australian Acoust. Soc., 12(1) (1984) 12-14.

3. Dunlop, J. I., Flexural vibrations of the triangle. Acustica, 55 (1984) 250-3.

4. Rossing, T. D., The acoustics of bells. American Scientist, 72 (1984) 440-7.

5. Rossing, T. D., (ed.), The Acoustics of Bells, Benchmark Papers in Acoustics Series, Van Nostrand Reinhold, New York, 1984.

6. Perrin, R., Charnley, T. \& de Pont, J., Normal modes of the modern English church bell. J. Sound Vibr., 90 (1983) 29-49.

7. Roderer, J. G., Introduction to the Physics and Psychophysics of Music, Springer-Verlag, New York, 1975.

8. Plomp, R., Aspects of Tone Sensation, Academic Press, London, 1976.

9. Fletcher, N. H., Nonlinear theory of musical wind instruments. Applied Acoustics, 30 (1990) 85-115.

10. Strand5 is produced by G + D Computing, Suite 307,3 Smail Street, Ultimo NSW 2007, Australia.

11. Mills, R. G. J., Tuning of bells by a linear programming method. J. Acoust. Soc. Am., 85 (1989) 2630-3.

12. Morse, P. M., Vibration and Sound, McGraw-Hill, New York, 1948; reprinted by Acoustical Society of America, Woodbury, 1976, pp. 151-62.

13. Press, W. H., Flannery, B. P., Teukolsky, S. A. \& Vetterling, W. T., Numerical Recipes, Cambridge University Press, New York, 1986, p. 39. 


\section{APPENDIX}

The propagation of transverse elastic waves along a thin rod is described by the well-known equation ${ }^{12}$

$$
\frac{\partial^{4} y}{\partial x^{4}}=-\frac{4 \rho \partial^{2} y}{E a^{2} \partial t^{2}}
$$

where $y$ is the displacement normal to the rod, $x$ is the coordinate measuring length along the rod, $\rho$ is the density and $E$ the Young's modulus of the rod material, and $a$ is the radius of the rod. This equation, appropriately supplemented for longitudinal motion as we show presently, describes the behaviour of each straight section of rod, and we simply require to impose appropriate conditions at the bends where two rods meet, and at the free ends. In this way we can achieve an analytic solution to this approximate representation of the real problem, in such a way as to allow simple and rapid calculation of the normal mode frequencies.

Inspection of eqn (Al) shows that its general solution can be written in the form

$$
y_{n}(x)=\alpha_{n} \cos k x+\beta_{n} \cosh k x+\gamma_{n} \sin k x+\delta_{n} \sinh k x
$$

where $k$ is the wave number, given from eqns (A1) and (A2) in terms of the angular frequency $\omega$ by

$$
k^{4}=\frac{4 \omega^{2} \rho}{E a^{2}}
$$

In eqn (A2), which refers to a section of rod labelled by the subscript $n$, the quantities $\alpha_{n}, \beta_{n}, \gamma_{n}$ and $\delta_{n}$ are constants, the values of which are determined by the boundary conditions at the two ends of this section of rod.

Equation (A2), of course, describes only displacements normal to the axis of the rod, and to complete the specification we must allow the possibility of displacement parallel to its axis. For the section $n$ of the rod we use the symbol $\varepsilon_{n}$ to denote this parallel displacement. We take each $\varepsilon_{n}$ to be constant along the length of the relevant rod, which means that we are ignoring the possibility of longitudinal waves in the rod material. This is physically justified, since the frequencies of the normal modes associated with these waves are much higher than those of the bending modes, and they are therefore outside the frequency range in which lie the modes we wish to tune.

This completes the geometrical description of the motion. Since there are 5 straight sections of rod, this implies 25 unknown coefficients. We note, however, that the pentangle has a plane of mirror symmetry, shown in Fig. 2, and this implies that the modes must be either symmetric or 
antisymmetric in relation to this plane. Applying this condition reduces the number of unknown coefficients to 15 .

These 15 coefficients are, however, not adequate to describe the dynamics of the problem. In particular, we require an appropriate balancing of forces and moments at the corners of the pentangle. The bending moments are properly described in terms of the elastic moduli, the rod radius, and the second derivative of the normal displacement $y$. The shear forces similarly involve the elastic moduli and the third derivative of the normal displacement. Description of the tension forces in the rod, however, requires introduction of tension forces $T$ which vary along the length of each rod. We are concerned only with matching conditions at the corners, and this is seen to introduce a further 5 independent quantities. These can be designated, with reference to Fig. 2, in terms of the symbol used for the corner (or the centre $\mathrm{O}$ ) and the rod number, as $T_{1}^{\mathrm{O}}, T_{1}^{\mathrm{A}}, T_{2}^{\mathrm{A}}, T_{2}^{\mathrm{B}}$ and $T_{3}^{\mathrm{B}}$. The tension at the free end $\mathrm{C}$ clearly vanishes. Symmetry considerations have been used to allow us to specify tensions for one half of the pentangle only.

Adding the 5 tension quantities to the 15 independent displacement parameters gives the total of 20 independent parameters necessary to specify the dynamics of the simplified thin-rod model. We now require 20 linearly independent equations relating these quantities. Once these have been written down and solved, we have a nonlinear equation in the wave number $k$, or equivalently in the frequency $\omega$, the solutions of which are the mode frequencies for the pentangle. We shall not go through this in exhaustive detail, but simply give the origin and general nature of the equations and show how they can be solved.

For each of the two bends $\mathrm{A}$ and $\mathrm{B}$, we require that the rods join together in a continuous manner ( 2 equations) and that their slopes $\partial y / \partial x$ match ( 1 equation), so that the bend angle is not distorted, for this would take an infinite moment about the join point. Furthermore, consideration of a tiny element of rod at the bend shows that, if its motion is to remain finite, the bending moments in the two rods at the join must be equal, implying continuity of $\partial^{2} y / \partial x^{2}$ ( 1 equation). Finally the forces exerted on the element by the two rods must balance in two orthogonal directions in the plane (giving 2 equations involving $\partial^{3} y / \partial x^{3}, T$, and the bend angle. This gives 6 equations at $\mathrm{A}$ and a further 6 at $\mathrm{B}$, making 12 in all.

At the free end $\mathrm{C}$, the bending moment and the shear force must both vanish, giving $\partial^{2} y / \partial x^{2}=0$ and $\partial^{3} y / \partial x^{3}$ (2 equations). We have already set the tension force equal to zero by not including it among the unknowns.

We then consider the longitudinal motion of each rod section under the influence of the difference between the tensions at its two ends. This 
is equal to the product of the mass and acceleration of the rod, and is thus proportional to the displacement quantity $\varepsilon$, the rod length, cross section and density, and the square of the frequency ( 3 equations).

This gives a total of 17 equations. The remaining 3 are derived for the point $\mathrm{O}$ on the symmetry plane, and are different for symmetric and antisymmetric modes. For the symmetric case, clearly $\partial y / \partial x=0$, and $\partial^{3} y / \partial x^{3}=0$, while the necessity for a stationary centre of mass requires that $T_{1}^{\mathrm{O}}=0$. For the antisymmetric case, symmetry dictates that $y=0$, $\partial^{2} y / \partial x^{2}=0$, and $\partial^{4} y / \partial x^{4}=0$. In either case we have 3 additional equations, making the total set up to the required number of 20 .

The 20 equations are homogeneous, since no external forces are involved, and the necessary and sufficient condition that they have a real solution is that the determinant of the matrix of their coefficients should vanish. This determinant is complicated, since the coefficients themselves are seen from (A2) to involve quantities such as $\cos k a$ and $\cosh k a$. Fortunately there are simple computer programs ${ }^{13}$ for evaluating a determinant once its elements are given numerical form by choosing a value of $k$, so that the problem reduces to using such a program to search for those values of $k$ for which the determinant vanishes. We can then use eqn (A3), with values of the elastic constants inserted, to convert these $k$-values to frequencies. A program to perform this operation is easily written and gives the first 6 or 7 mode frequencies to good precision in a few minutes on an AT-compatible microcomputer using Microsoft QuickBasic. Indeed the speed can be further improved by first using algebraic manipulation to reduce the rank of the determinant. There is no problem about requiring extra constraints and eliminating rigid-body modes as there is in some implementations of the corresponding finite-element calculation. 